\title{
Failure mode analysis and damage control for jacket platform structures
}

\author{
Ping $\mathrm{Li}^{1, \mathrm{a}}$, Hong Lin ${ }^{1, \mathrm{~b}}$, Lei Yang ${ }^{2, \mathrm{c}}$, Yu Zhang ${ }^{1}$, and Bao Qi ${ }^{1}$ \\ ${ }^{1}$ College of Pipeline and Civil Engineering, China University of Petroleum, Qingdao 266580, China \\ ${ }^{2}$ College of Science, China University of Petroleum, Qingdao 266580, China \\ a m18306489964_1@163.com, 'binhong@upc.edu.cn, 'Y Yang_lei_famous@126.com
}

Keywords: Jacket structure; Failure mode analysis; Internal force redistribution; FEM; Damage control

Abstract. Jacket offshore platform structure are the big space structures usually used in field of offshore oil engineering. Local damage of platform may occur in the action of emergency, and will result in the whole failure of platform under extreme loads. So it is necessary to analyze the failure mode and damage control method for the jacket platform. We first studied the failure modes of jacket test model under different loads by experiments. Then combined with FEM software ANSYS, the internal force redistribution of jacket structure in the process of failure is analyzed. Further, we studied the damage control methods for jacket upper leg, and analyzed the reinforcement effect.

\section{Introduction}

The large offshore steel structure of jacket platform is different from that of the large steel structure on the land and it is mainly reflected as follows: the structure is more complex, the volume is bigger, the technology content is higher, the cost is higher, the marine environment is more bad and so on [1]. According to incomplete statistics, since the 1970s, the fixed platform for the destruction of the accident reached about 240 around the world and this caused hundreds of casualties. For example: accident [2] happened in the UK north sea Piper Alpha platform, EI-322A platform due to the effect suffered Lili storm, the EI-322A platform [3] was damaged and eventually retired.

In order to reduce the probability of the occurrence of such accidents, and reduce the harm to the marine environment caused by offshore platform, offshore platform need to maintain a higher ability to resist collapse $[4,5]$ and a good safety performance. So it is very important to study the collapse mechanism [6,7,8,9] of jacket platform and the failure mode [10] of intact platform and the corresponding damage control method of intact platform. In this paper, we analyzed the failure modes of jacket structure under different loads and studied the damage control corresponding methods [11] .

\section{Experimental Study}

\section{Experiment equipment and loading conditions}

The loading device is designed and loading in both vertical and horizontal, as shown in figure 1-1.

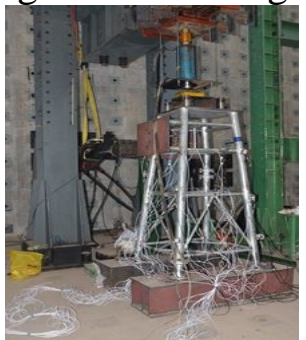

Fig. 1-1 Experimental loading

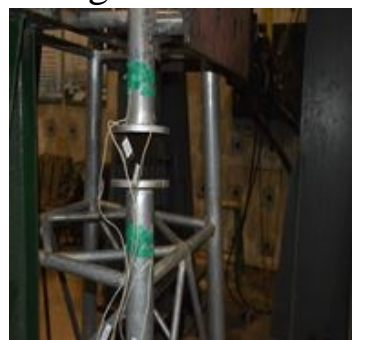

Fig. 1-2 Remove element 6 and remove element 9

\section{Internal force redistribution under vertical load}

Under the vertical load, the structure damage is simulated with the way in which the flange plate is removed. When the load increases from 0 to $6 \mathrm{t}$, the structure strains is measured in three cases where the structure is complete, the upper leg flange is removed and the lower flange plate is removed respectively and the condition of the distribution of the internal force is analyzed. The loading 
experiment schematic of the damaged structure is illustrated in figure 1-2. There are 25 strain gauge points on the model.

The strain value of each measuring point is measured during the experiment and we compared the measurement values of the intact structure with that of the damaged structure. It is found that the value of a few points about intact structure of several measuring point and after the demolition of damaged members of measuring point are apparent contrast, drawing No. 6, 13, 14, 16 load -micro strain curve shown in figure 1-3. From figure 1-3, we can see that the redistribution of internal force is obvious and the damage to pile leg has a greater impact on the overall structure.
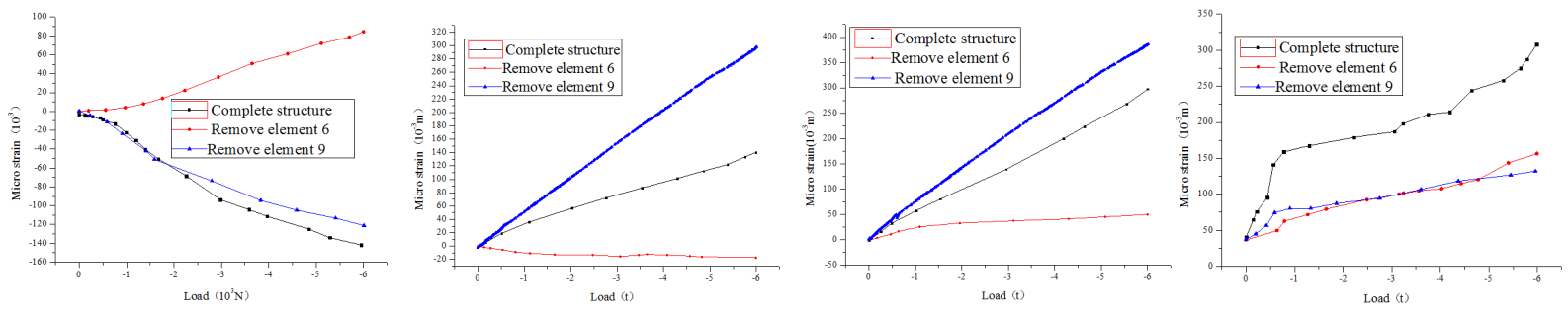

Fig. 1-3 Four key points of load-micro strain curve

\section{Experimental results under combined loads}

Under the combined action of vertical and horizontal loads, the experiment for intact jacket is carried out again. Under the vertical load of two tons, the horizontal load is gradually increased, when the upper jacket structure has obvious deformation, meanwhile, when the horizontal displacement reaches the height of the model $1 / 20$ that is $0.09 \mathrm{~m}$, the platform has been a failure, as shown in figure $1-4$, we stopped the experiment at once. The load -displacement curves is measured during the experiment, as shown in figure 1-5.

Through the the experimental results, it is concluded that the failure mode of intact jacket structure under the combined action of the dead load and vertical load and horizontal load is a large deformation in the upper of the jacket leg.

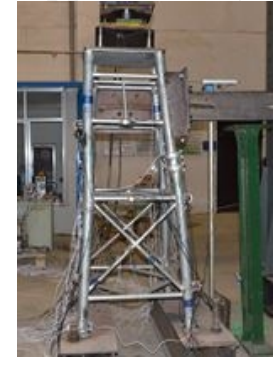

Fig. 1-4 Failure mode Fig. 1-5 Load-displacement curve

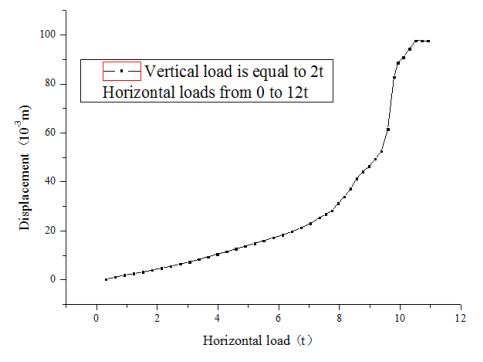

Fig. 2-1 Element number and leg number

\section{Finite element simulation}

\section{The establishment of finite element model}

The experimental model is $1805 \mathrm{~cm}$, which is $1 / 20$ of the actual model. The finite element model are modeled by PIPE20 element and PLANE182 element. The finite element model can be seen from the figure 2-1, and it has 84 nodes, 102 elements.

\section{Internal force redistribution under vertical load}

The loads of the jacket are the dead weight and the load from 0 tons to 6 tons, on the upper part of four nodes are evenly arranged 6 load steps, each load step increases the force of 1 tons. Four pile legs are for all constraints. We respectively simulate the intact structure model and the damaged structure model by ANSYS. The axial strain of four measuring points in the intact structure and damaged structure under different loads are extracted respectively. As the number 14 and number 16 are symmetrical, so we only draw the first three points of the load-strain curves, as shown in figure 2-2. 

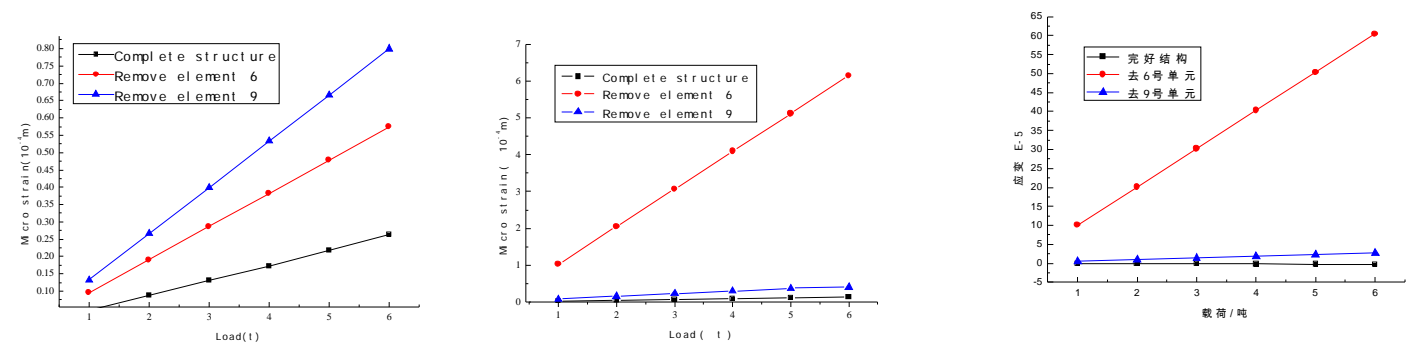

Fig.2-2 No. 6,No. 13,No. 14 measurement point

See from figure 2-2, the results of the finite element simulation of each measuring point are different from the experimental data of each measurement point, but the overall trend is the same and the damage component has great influence on the redistribution of internal force of the whole structure. In order to better reflect the redistribution of internal force, we extracted the axial force and bending moment before and after removing element 6 . As shown in figure 2-3. Internal force redistribution phenomenon can be clearly seen from the figure 2-3.

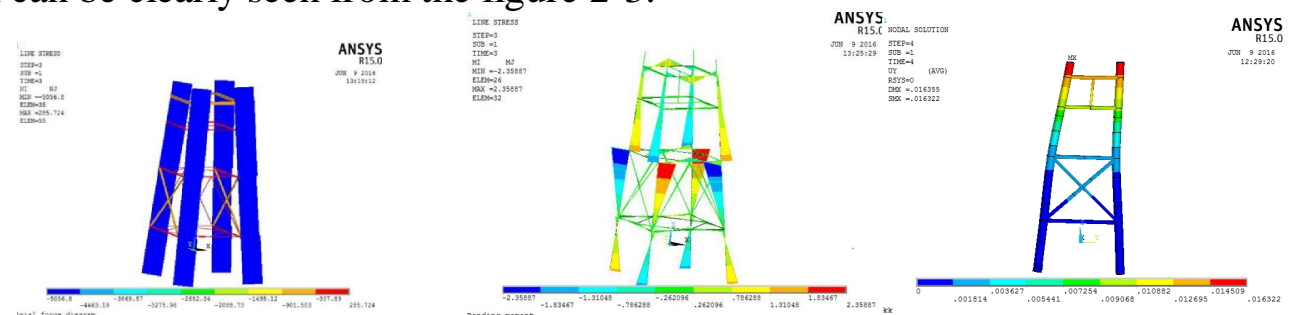

Fig. 2-3 Axial force before and after removing element 6 Fig. 2-4 Deformation

\section{The failure mode of the jacket under composite load}

Under the horizontal and vertical loads on the intact jacket structure, ANSYS numerical simulation is carried out. The load is set as follows: taking into account both dead weight and vertical load, the vertical load is simplified as a 2 tons force at the top of the four pile legs and remains unchanged. Horizontal load of the four nodes on the first and the two layers of the jacket type $\mathrm{X}$ is along the $\mathrm{Y}$ direction. When the horizontal load reaches 32 tons, the highest point of horizontal displacement reach $1 / 20$ of the height, deformation diagram is shown in figure $2-4$, and the failure mode is the yield in the upper leg of the structure.

\section{Failure analysis of damaged structure}

\section{Experimental study}

In order to analyze the failure mode of damaged structure, flange plate at the lower end of the 1 leg is removed and the experiment is carried out as shown in figure 3-1. The experiment is carried out under the condition that vertical load is 2 tons and horizontal load is increased gradually. Strain and displacement of the platform structure are measured in the experiment. As can be seen from figure 3-2, when the horizontal load is increased to 8.6 tons, the $\mathrm{K}$ type of the diagonal brace member has a clear out of plane instability, resulting in a larger lateral displacement of the entire jacket.

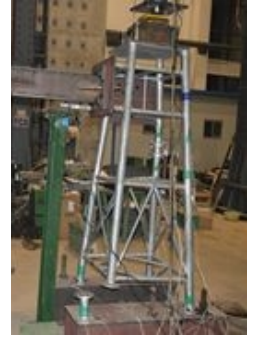

Fig. 3-1 Structure loading

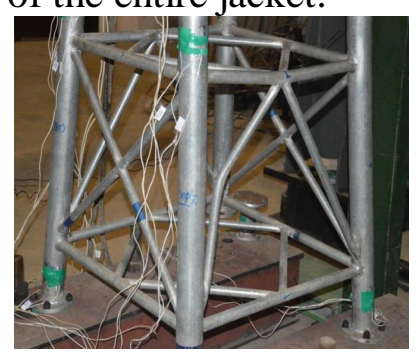

Fig. 3-2 Structure failure mode 


\section{Numerical simulation of damaged structure}

After removal of unit 6, we draw the stress contour plot. And as can be seen from figure 3-3, the brace of $\mathrm{K}$ type will buckle. Then we carry out the buckling analysis both by theoretical calculation and ANSYS software. The theoretical critical pressure can be obtained by the Euler formula 3-1 [12].

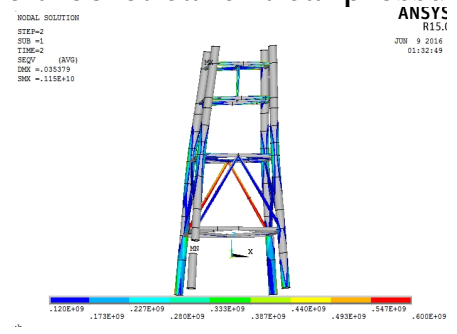

Fig. 3-3 Stress contour plot

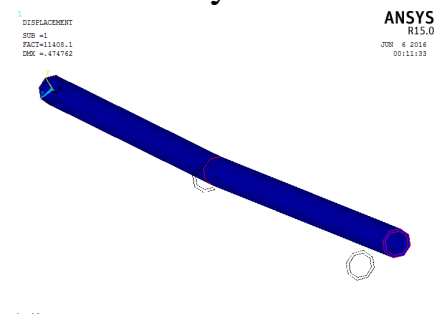

Fig. 3-4 Eigenvalue buckling analysis

$$
P_{l j}=\frac{\pi^{2} E I}{(\mu L)^{2}}
$$

\section{3-1 )}

Where, $\mu l$ as the equivalent length, $\mu$ for length coefficient, $\mu=0.5$. We take the parameters of this section into the formula, the critical pressure of the theory can be obtained $184613.25 \mathrm{~N}$.

Then we carry out the finite element eigenvalue buckling analysis by ANSYS. The constraint is set as one end fixed and the other end is free. The calculated results are shown in figure 3-5. The critical pressure to be simulated by figure 3-5 is $11408.1 \mathrm{~N}$. We take the parameters into the formula (3-1), $\mu=2$, it is concluded that the effective length of the rod is $2 * 0.074254 \mathrm{~m}$. The theoretical critical pressure is $11541.7 \mathrm{~N}$. The relative error is $1.1 \%$. The correctness of the finite element simulation is proved.

\section{Reinforcement and its effect analysis of jacket structure}

In the course of the experiment, the component is damaged and reinforcement of the steel plate is adopted, as shown in figure 3-5. The reinforcement rib is set as the BEAM188 element. Rib provided with different thickness and shape, we can get different reinforcement effect.We use coupling method to deal with the contact part. The established model is shown in figure 3-6.

In order to further verify the effectiveness of the method, in the ANSYS, we mainly consider displacement of the highest point and internal force of the changes to simulate the reinforcement model.
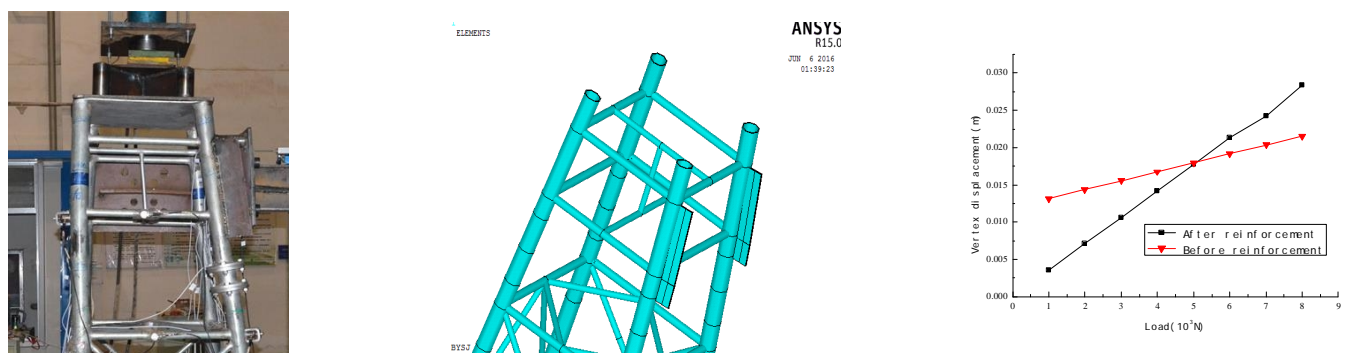

Fig. 3-5 Rib reinforcement Fig. 3-6 Rib reinforcement model Fig. 3-7 Displacement-load curve

We extract the top of node's displacement and draw from 1 to 8 tons of load-displacement curves, as shown in figure 3-7. As can be seen from figure 3-7, only when the load reaches a certain value, the reinforcement effect is very obvious. But in the whole loading process, after reinforcement, the load-displacement curve slope is decreases, it is indicated that the displacement with increase of the load is getting to slow down, which shows that the rib reinforcement for jacket structure is effective. 


\section{Conclusions}

(1) Under the action of vertical load and horizontal load, the jacket structure is inclined. Through experiment and ANSYS simulation, the main force bearing components can be obtained as leg and braces for offshore platforms. The diagonal brace buckling deformation is the failure mode of damaged structure.

(2) Rib reinforcement method can be used for repair and reinforcement of the damaged components. By experiments and finite element simulation, we can conclude that the rib reinforcement can slow down the damage of the structure.

(3) Under the action of vertical load, failure of the damage component leads to redistribution of internal force of structure. Closer to the damaged component, more obvious of the internal force redistribution.

\section{Acknowledgements}

This work was financially supported by National Natural Science Foundation of China (51209218), the Fundamental Research Funds for the Central Universities (15CX05065A, 15CX05003A) and Applied Basic Research Projects (Youth special) of Qingdao (14-2-4-58-jch).

\section{References}

[1] Shunfeng Gong. Study on structural impact damage and reliability and fatigue life assessment of offshore platform [D]. Zhejiang University, 2003

[2] Yi Lu. Alfa drilling platform big bang [J]; labor protection; 200406 period

[3] Xiao Dong . A series of big bang [J] in the UK; security and disaster prevention in Hunan; 200808 period

[4] Fan Xia. The collapse of jacket offshore platform [D]. Wuhan University of Technology, 2012

[5] Benrui Zhu, Guoming Chen, Hong Lin, et al. Numerical simulation of the whole process of collapse of jacket platform under strong typhoon [J]. ocean engineering, 2015, 33 (2).

[6] Junrong Wang . Correction method and Research on the model of structural damage detection of offshore platform [D]. Ocean University of China, 2009

[7] M.T.Vannan.H.M.Thompson and J.J.Griffin.An Automated Procedure for Platform Strength Assessment [C].Offshore .Technology Conference Houston Texas , 1994

[8] Lalani M,Shulteworth E P .The ultimate limit state of offshore platform using reserve and residual strength principles,Paper OTC 6309 ,Offshore Technology Conference. Txas, 1990

[9] Ueda Y.Plates and stiffened plate units of the idealized structural unit method [C].The Socitey of Naval Architects of Japan, 1990

[10] Xiaojiang Shang, Feng Qiu. Advanced analysis method and example application of structural finite element method (Third Edition) [M]. Beijing: China water resources and Hydropower Publishing House.2015.1

[11] Zhen Zhang. Study on hydraulic expansion grouting clamp device of offshore platform [D]. Harbin Engineering University, 2013

[12] Yingmin Lv . Mechanics of materials [M]. China University of Petroleum press, 2007.2 